\title{
High-Power VLF Transmission Experiment in the Radiation Belts: Initial Result from the DSX Mission
}

\section{Paul Song ( $\nabla$ paul_song@uml.edu ) \\ University of Massachusetts Lowell \\ Jiannan Tu}

University of Massachusetts Lowell https://orcid.org/0000-0002-6681-8759

Ivan Galkin

University of Massachusetts Lowell

James McCollough

Albuquerque

Gregory Ginet

MIT

William Johnston

Air Force Research Laboratory

Yijun Su

Air Force Research Laboratory

Michael Starks

Air Force Research Laboratory

Bodo Reinisch

University of Massachusetts Lowell

Umran Inan

Stanford University

David Lauben

Stanford University

Ivan Linscott

Stanford University

William Farrell

NASA Goddard Space Flight Center

Shawn Allgeier

MIT Lincoln Laboratory

Richard Lambour

MIT Lincoln Laboratory

Jon Schoenberg

MIT Lincoln Laboratory

William Gillespie 
MIT Lincoln Laboratory

\section{Stephen Stelmash}

University of Massachusetts Lowell

Kevin Roche

University of Massachusetts Lowell

Andrew Sinclair

Air Force Research Laboratory

Jenny Sanchez

Air Force Research Laboratory

\section{Article}

\section{Keywords:}

Posted Date: February 4th, 2022

DOI: https://doi.org/10.21203/rs.3.rs-1261412/v1

License: (c) (1) This work is licensed under a Creative Commons Attribution 4.0 International License. Read Full License 


\section{Abstract}

Space weather phenomena threaten the space assets that bring us services via space technologies, such as the Global Positioning System, communication systems with satellite relays, and most global TV broadcast networks, which have provided unprecedented convenience to everyday life and opportunities to businesses. A hazard among phenomena 1 is the population of relativistic electrons in the region called Van Allan radiation belts2. These electrons can be trapped for years once produced by either natural3 or artificial processes 4 and can damage the electronics and degrade the solar panels on satellites. Intense investigations have begun with recently launched NASA satellites, Van Allen Belt Probes A and B in 20125-13. To remedy the threat and reduce the resulting damage, artificial processes can be introduced to shorten the lifetime of these particles 14 with mechanisms such as pitch-angle diffusion through waveparticle interaction 15-17 by transmitting very-low-frequency (VLF) waves into radiation belts. To directly transmit the VLF waves in space is an extremely challenging task, and previous theoretical and numerical predictions of the radiation impedance differ more than five orders in magnitude18-23. Here we show the measurements of radiation impedance from high-power VLF wave transmission experiments in the radiation belts to help settle the dispute of the previous studies. The measured radiation reactance disagrees with the most influential theoretical model18,19,22 and the vacuum model, but proves the plasma sheath model and simulation of the antenna-plasma interaction20,21,23. A new discovery is that the measured radiation resistance decreases as the transmission frequency increases. Our results demonstrate the possibility to transmit high power in space and validated the design and technology for further high-power space-borne VLF transmitters. The physical understanding obtained in this study will also provide a guide to laboratory whistler mode wave injection experiments 24 , especially in controlled fusion25.

\section{Introduction}

Space weather phenomena threaten the space assets that bring us services via space technologies, such as the Global Positioning System, communication systems with satellite relays, and most global TV broadcast networks, which have provided unprecedented convenience to everyday life and opportunities to businesses. A hazard among phenomena ${ }^{1}$ is the population of relativistic electrons in the region called Van Allan radiation belts ${ }^{2}$. These electrons can be trapped for years once produced by either natural ${ }^{3}$ or artificial processes ${ }^{4}$ and can damage the electronics and degrade the solar panels on satellites. Intense investigations have begun with recently launched NASA satellites, Van Allen Belt Probes A and B in $2012^{5-13}$. To remedy the threat and reduce the resulting damage, artificial processes can be introduced to shorten the lifetime of these particles ${ }^{14}$ with mechanisms such as pitch-angle diffusion through waveparticle interaction ${ }^{15-17}$ by transmitting very-low-frequency (VLF) waves into radiation belts. To directly transmit the VLF waves in space is an extremely challenging task, and previous theoretical and numerical predictions of the radiation impedance differ more than five orders in magnitude ${ }^{18-23}$. Here we show the measurements of radiation impedance from high-power VLF wave transmission experiments in the radiation belts to help settle the dispute of the previous studies. The measured radiation reactance 
disagrees with the most influential theoretical model $18,19,22$ and the vacuum model, but proves the plasma sheath model and simulation of the antenna-plasma interaction ${ }^{20,21,23}$. A new discovery is that the measured radiation resistance decreases as the transmission frequency increases. Our results demonstrate the possibility to transmit high power in space and validated the design and technology for further high-power space-borne VLF transmitters. The physical understanding obtained in this study will also provide a guide to laboratory whistler mode wave injection experiments ${ }^{24}$, especially in controlled fusion $^{25}$.

\section{Methods}

The U.S. Air Force's Demonstration and Science Experiments (DSX) satellite was launched on June 25, 2019 , into an orbit that passes through the radiation belts to study wave-particle interaction processes ${ }^{26}$. On the satellite is a transmitter with an 82-meter tip-to-tip dipole antenna. In order to transmit high power, the novel transmitter, named Transmitter-Narrowband receiver-Tuner (TNT), carries a tuner that is able to automatically tune to a desirable resonance frequency according to the plasma conditions as the satellite moves rapidly in space. After the successful deployment of the antenna in July 2019, TNT conducted a large number of successful transmission experiments. The mission completed on May 31, 2021.

Figure 1 shows (a) the DSX structure and (b) the TNT equivalent circuit. In TNT, the Digital Control Unit (DCU) generates the time-series of desirable frequency and waveform. This signal is sent to two TunerAmplifier-Transmission Units (TATU), denoted by subscripts + and - , respectively, and is amplified to $V_{1 \pm}$. Subscript 1 denotes the fundamental frequency. Each TATU, in addition to the power amplifier, consists of an adaptive tuner with a bank of inductors, $L_{1}$ with internal resistance $R_{1}$, and capacitators $C_{1}$. The signal, after transiting the tuner, is then fed into the antenna. The voltage at each antenna terminal relative to the ground of TATU is measured. In this study, the two TATUs are set with $180^{\circ}$ phase difference, i.e., . TNT is also able to transmit the third harmonics with the correct phase and amplitude so that the wave form can mimic a square-wave. In this experiment, since the radiation impedance is a function of space plasma condition, TNT is set at a given $L_{1}, R_{1}$, and $C_{1}$ and transmits first in a large frequency range with coarse frequency step, aiming at finding the frequency in which the TATU and plasma condition are tuned, and then successively reduces the frequency range for a refined tuning condition. From each curve of antenna voltage as a function of frequency three quantities are derived: the tuning resonance frequency, $f_{\mathrm{r}}$, where the voltage reaches maximum, peak voltage value, $V_{\mathrm{a}}$, and half-power frequency width, $\Delta f$, which is the frequency span between two points where the voltage drops to $1 / \sqrt{ } 2 V_{a}$ (i.e., at half-power). The resonance occurs when the frequency satisfies $\left(2 \pi f_{r}\right)^{2}=1 / L C$ where $L$ and $C$ are the total equivalent inductance and capacitance of an R-L-C circuit. The quality factor $Q=f_{r} / \Delta f=2 \Pi f_{r} L / R$ of the circuit is derived, where $R$ is the total resistance. From Figure $1 \mathrm{~b}$, the antenna reactance $X_{\mathrm{a}}$, capacitance, $C_{\mathrm{a}}$, resistance, $R_{\mathrm{a}}$, and power delivered to the antenna, $P_{\text {out }}$, can be determined with the system and measured parameters 
$-X_{a}=1 / 2 \pi f_{r} C_{a}=\frac{2 \pi f_{r} L_{1}}{1-\left(2 \pi f_{r}\right)^{2} L_{1} C_{1}}$

$R_{a}=\left(\frac{2 \pi f_{I} L_{1}}{Q}-R_{1}\right)\left(1+\frac{C_{1}}{C_{a}}\right)^{2}$

$P_{\text {out }}=\begin{array}{ll}1 & V_{a}^{2} R_{a} \\ 2 & R_{a}^{2}+X_{a}^{2}\end{array}$

where $L_{1}=L_{1+}+L_{1-}, C_{1}=C_{1+} C_{1-} /\left(C_{1+}+C_{1-}\right), R_{1}=R_{1+}+R_{1-}$,

$C_{a}=C_{a+} C_{a-} /\left(C_{a_{+}}+C_{a_{-}}\right)$, and $V_{a}=V_{a_{+}}+V_{a_{-}}$. Figure 2 shows an example of measured resonance curves for each of the three operation frequency bands on the same day inside the radiation belts. The driving voltage of both TATU branches $V_{1 \pm}$ is the same, $88 \mathrm{~V}$, for the three bands. The antenna peak voltage increases to approximately $Q$ times $V_{1 \pm}$. The lower band has a broader resonance curve and hence a smaller $Q$.

\section{Results}

In the 21-month experiment, a total of 488,830 resonance curves have been collected from each antenna branch. About $39 \%$ of them are when one of the antenna branches were turned off during experiment or have more complicated features than a single peak between half-power points or one of the half-power points is out of frequency range of the specific band, so that the $Q$-factor cannot be meaningfully determined. About another $6 \%$ of the total involved transmission of the third harmonics which may interfere with the determination of the fundamental frequency in this study. There are also some anomalous cases, about $9 \%$, when the resonance frequency and bandwidth from two antenna branches are very different and different from neighboring measurements. After excluding those three types of events, a total of 231,920 resonance curves are available from each of the two branches.

Because the most dangerous high energy electrons with long lifetime are trapped in the region called plasmasphere ${ }^{2}$, for the purpose of remediation of radiation belts, we are most interested in the transmission in the plasmasphere. We use the Carpenter-Anderson plasmaspheric model ${ }^{27}$ to select the transmission events in the plasmasphere. Furthermore, because the radiation impedance most likely depends on the transmission voltage and we are most interested in high-power transmission, in this initial report, we focus on events with $V_{1 \pm}$ greater than $80 \mathrm{~V}$, i.e., peak-to-peak voltage of greater than $160 \mathrm{~V}$. There are about 166,300 resonance curves satisfy these conditions and are shown in Figure 3 . The data are binned with $100 \mathrm{~Hz}$ in frequency and 80 bins for each of the three parameters, $-X_{\mathrm{a}}, R_{\mathrm{a}}$, and $P_{\text {out }}$. The color coding presents the relative number of the events in each frequency and parameter bin-cell.

The overall trends for $-X_{\mathrm{a}}$ and $R_{\mathrm{a}}$ are clear-decreasing when frequency increases both within each band and across the three bands. A power-law fit shows that $-X_{a} \propto f_{r}^{-0.91}$ and $R_{a} \propto f_{r}^{-1.73}$. On the other hand, $P_{\text {out }}$ decreases within each band but increases when going to higher bands. Within each band 
$P_{\text {out }} \propto f_{r}-2.09$. In the upper band, the radiated power can be as large as $50 \mathrm{~W}$, if the antenna dissipation and local wave-particle resonance absorption are weak.

To understand the results, we plot several theoretical model results on top of the observation in Figure 4. Dashed lines are from a vacuum model, solid lines represented Wang and Bell model ${ }^{19}$, and black patches of plus signs from two Song et al. models ${ }^{20,23}$. The vacuum reactance $X_{\mathrm{a}}$ has a similar trend to that in plasma but about a factor 3 larger. The radiation resistance is many orders of magnitude greater than that in vacuum with a distinctly different trend. Therefore, the vacuum model may be irrelevant to VLF transmission in plasma.

\section{Discussion}

Wang and Bell ${ }^{19}$ developed a quantitative model of the reactance and resistance for whistler mode transmission in plasma, solid lines in Figure 4. Chevalier et al. ${ }^{22}$ conducted a computer simulation, in open circles, as a part of DSX mission development. The two results agree well with each other. However, both appear to be in conflict with the measurements, especially when noting that their reactance in the upper panel has been multiplied by a factor of 3,000 in order to show them on the plot. Furthermore, in addition to the difference in the magnitude, the overall trend as a function of frequency is in a wrong direction. In their frequency range, the antenna resistance is more than an order of magnitude smaller than the measurements. Therefore, we conclude that Wang and Bell ${ }^{19}$ and Chevalier et al. ${ }^{22}$ models are incompatible with the experiment.

Song et al. ${ }^{20}$ developed a physical model of the plasma sheath surrounding a VLF transmission antenna as part of TNT development study. They reasoned that when the antenna is charged with alternately varying high voltage during transmission, charged particles will move between the two antenna branches in response. When the electromagnetic field oscillates in the whistler mode frequency range, electrons will be repelled from the negatively charged branch of the antenna and attracted to the positively charged one while the ions do not have enough time to respond before the field reverses. Around the negatively charged branch, an ion sheath is formed with net positive charge. Around the positive branch, on the other hand, there is a tendency to form an electron sheath with net negative charges. The overall effect of this process is that the sheath is formed around each branch of the antenna with an oscillating radius, which is achieved mostly by electron movements between the two branches. The corresponding electric current of such electron movements tends to cancel the driving current of the antenna, which produces the difficulty for whistler mode transmission in plasma. The equivalent reactance of the sheath is

$$
-X_{a}=\frac{1}{\alpha 2 \Pi^{2} f \varepsilon_{0} l}\left[\ln \left(\frac{I_{a}}{\Pi^{2} f l e N_{0} r_{a}^{2}}+2\right)-1\right]
$$


where $f$ is the transmission frequency, $l, e, N_{0}, r_{a}, \varepsilon_{0}$, and $I_{a}$ are the antenna length, elementary electric charge, plasma density, antenna radius, permittivity in vacuum, and antenna current. We have introduced a factor $a$ to Song et al. ${ }^{20}$ to account for the effect of the antenna structure. The TNT antenna consists of three separate parallel conducting wires, for the purpose of redundancy and robustness, of $0.15 \mathrm{~mm}$ each in radius along a circle of diameter of $20 \mathrm{~cm}$. In a highly simplified model, if each wire has a capacitance $C$, the three parallel wires could be considered as three capacitors in parallel and the total capacitance would be $3 \mathrm{C}$. However, the three wires may have some interaction and the net capacitance could be represented as $a C$ and $a$ can be estimated during the antenna development. Experiments have indicated $a=2.2$ in vacuum of such a three-wire antenna. According to (2), the antenna reactance depends on the plasma density logarithmically. We assume that the plasma density is $2,000 / \mathrm{cc}^{28}$ based on measurements from the RPI instrument on the IMAGE spacecraft. The prediction is shown as black patched of plus signs in the upper panel of Figure 4. Because they are so close together, they form black patches. The model appears to be generally consistent with the measurements.

Also, during the TNT development Song et al. ${ }^{23}$ developed a model of whistler mode radiation resistance. It is based on the Huygens-Fresnel diffraction theory to derive the radiation from an antenna. The model predicts that the radiation resistance is

$$
R_{\text {rad }} \approx \frac{3 \Pi^{3} \mu_{0}}{c} f_{c e}^{3} f_{p e} f^{-2} d^{2}=3.9 \times 10^{-13} f_{c e}^{3} f_{p e} f^{-2} d^{2}
$$

3

where $\mu_{0}, c, f_{p e^{\prime}} f_{c e^{\prime}}$ and $d$ are permeability of free space, the speed of light, electron plasma frequency, electron gyrofrequency, and half-length of antenna, respectively. For typical conditions of DSX, $R_{\mathrm{rad}}$ is 2.6 $\mathrm{k} \Omega$ at $10 \mathrm{kHz}$ and is proportional to $f^{-2}$. The black patches of plus signs in the lower panel of Figure 4 show the prediction of the model. Because the measures of impedance, $X_{\mathrm{a}}$, and $R_{\mathrm{a}}$, are consistent with the theoretical predictions given in (2) and (3), we conclude that the antenna sheath model ${ }^{20}$ and whistler mode radiation model ${ }^{23}$ approximate the process in reality.

Furthermore, Tu et al. ${ }^{21}$ conducted full particle simulations to investigate plasma sheath structures around a VLF antenna in plasma. In their simulation, dipole antenna with tip-to-tip length of $200 \mathrm{~m}$ and radius of $0.2 \mathrm{~m}$ is used. The resulting reactance is scaled based on Song et al. ${ }^{20,23}$ and is plotted as a triangle on the top panel of Figure 4. From the simulated phase difference between the antenna voltage and current, the antenna resistance is calculated and plotted as a triangle in the bottom panel of Figure 4 . Both reactance and resistance from Tu et al. ${ }^{21}$ are reasonably consistent with the DSX measurements.

The net antenna power output is more complicated by several effects, such as tuner setting and plasma conditions. From (1) and Figure 1b, it can be approximated as 


$$
P_{\text {out }} \approx \frac{V_{1}^{2}}{2} \frac{R_{a}}{\left(R_{a}+R_{1}\right)^{2}}
$$

\section{4}

The approximation neglects the current flowing through $C_{1}$. In the experiments, each of the tuning bands uses a fixed inductance and increases capacitance $C_{1}$, corresponding to transmission from higher frequency end to lower frequency end within each band. From the resonance condition, the lower frequency band uses a greater $L_{1}$ and hence greater $R_{1}$ as the two are proportional. Since $V_{1}$ for the data included in Figure 3 varies in a relatively small range, the step-like increase in $P_{\text {out }}$ from the lower band to higher band is due to the decrease in $L_{1}$ and hence $R_{1}$ in each band setting. Within each band, the change in $R_{1}$ is relatively small, but $R_{\mathrm{a}}$ decreases with frequency as frequency increases, resulting in the power decrease within each band.

\section{Summary}

In summary, the DSX-TNT with 82-m tip-to-tip antenna has been used to conduct successfully a series of high-power VLF transmission experiments. The radiated power can be as high as 50W. To put it in perspective, the Radio Plasma Imager (RPI) transmitter ${ }^{29,30}$ on the NASA IMAGE satellite also transmitted in the VFL frequency range. When scaled to DSX antenna length, the radiated power would have been $0.25 \mathrm{~W}$. In the plasmasphere, for the electrically-short antenna, the antenna reactance is much greater than its resistance and the capacitance is greater than that of vacuum. The capacitance is well described by (2) indicating that the sheath capacitance may dominate the antenna reactance. It is consistent with Tu et al. ${ }^{21}$ simulation result although it is fundamentally inconsistent with the Wang and Bell ${ }^{19}$ model and Chevalier et al. ${ }^{22}$ simulation. The observed resistance, proportional to $1 / f_{r}^{2}$, is fundamentally different from that in the vacuum for a short electrical dipole antenna and is consistent with the prediction of Song et al. ${ }^{23}$ The rapid decrease of resistance with frequency is a discovery from the experiment because, according to the picture for vacuum, in higher frequencies, the ratio of the antenna length to the wavelength increases and should have led to a greater radiation resistance. This result will inform future development of space-based VLF capabilities and also whistler wave injection experiments for controlled fusion.

\section{References}

1. Song, P., H. J. Singer, G. L. Siscoe, Space Weather, Geophys. Mon., vol. 125, AGU, DC., (2001)

2. Russell, C. T., J. G. Luhmann, R. J. Strangeway, Space Physics: An Introduction, Cambridge University Press, (2016)

3. Green, J. L., S. Boardsen, L. Garcia, W. W. L. Taylor, S. F. Fung, B. W. Reinisch, On the origin of whistler mode radiation in the plasmasphere, J. Geophys. Res., 110, A03201, 
https://doi.org/10.1029/2004JA010495, (2005)

4. Albert, J. M., D. H. Brautigam, R. V. Hilmer, and G. Ginet, Dynamic radiation belt modeling at air force research laboratory, in Space Weather, 281, eds., P. Song, H. J. Singer, and G. L. Siscoe, Geophys. Mon., vol. 125, AGU, DC., (2001)

5. Reeves G D, Spence H E, Henderson M G, Morley S. K., Friedel R H W, et al., Electron Acceleration in the Heart of the Van Allen Radiation Belts, Science, 991 - 994 DOI: 10.1126/science.1237743 (2013)

6. Baker D N, Kanekal S G, Hoxie V C, Henderson M G, Li X, et al. A Long-Lived Relativistic Electron Storage Ring Embedded in Earth's Outer van Allen Belt, Science, 186-190 DOI: 10.1126/science.1233518, (2013)

7. Hudson M K, Space physics: A fast lane in the magnetosphere, Nature, 383-384, DOI: 10.1038/504383a, (2013)

8. Mozer F, Bale S., Bonnell J W, Chaston C., Roth I, et al. Megavolt Parallel Potentials Arising from Double-Layer Streams in the Earth's Outer Radiation Belt, Physical Review Letters DOI: 10.1103/PhysRevLett.111.235002, (2013)

9. Sprits Yuri Y, Subbotin Dmitriy, Drozdov Alexander, Usanova Maria E., Kellerman Adam, et al., Unusual stable trapping of the ultrarelativistic electrons in the Van Allen radiation belts, Nature Physics, 699 703 DOI: 10.1038/nphys2760, (2013)

10. Thorne R M, Li W, Ni B, Ma Q, Bortnik J, et al., Rapid local acceleration of relativistic radiation-belt electrons by magnetospheric chorus, Nature, 411 - 414, DOI: 10.1038/nature12889, (2013)

11. Turner D. L, Space science: Near-Earth space shows its stripes, Nature, 308 - 309, DOI: 10.1038/507308a, (2014)

12. Ukhorskiy A Y, Sitnov M I, Mitchell D G, Takahashi K, Lanzerotti L J, et al, Rotationally driven 'zebra stripes' in Earth's inner radiation belt, Nature, 338 - 340 DOI: 10.1038/nature13046, (2014)

13. Breneman A. W., Halford A., Millan R., McCarthy M., Fennell J, et al., Global-scale coherence modulation of radiation-belt electron loss from plasmaspheric hiss, Nature, 193 - 195 DOI: 10.1038/nature14515, (2015)

14. Stone, R., U.S. tests ways to sweep space clean of radiation after nuclear attack, Science, doi:10.1126/science. aba7112, Dec 26, (2019)

15. Kennel, C. F., H. E. Petschek, Limit on stably trapped particle fluxes, J. Geophys. Res., 71, 1, (1966)

16. Abel, B., and R. M. Thorne, Electron scattering loss in the Earth's inner magnetosphere: 1. Dominant physical processes, J. Geophys. Res., 103, 2385, (1998)

17. Thorne, R. M., B. Ni, X. Tao, R. B. Horne and N. P. Meredith, Scattering by chorus waves as the dominant cause of diffuse auroral precipitation, Nature, 467, 943-946, doi:10.1038/nature09467, (2010)

18. Balmain, K. G., The impedance of a dipole short antenna in a magnetoplasma, IEEE Trans. Antennas Propag., AP-12 (5), 605-617, (1964) 
19. Wang, T. N., and T. F. Bell, On VLF radiation resistance of an electric dipole in a cold magnetoplasma, Radio Science, 5, 605, (1970)

20. Song, P., B. W. Reinisch, V. Paznukhov, G. Sales, D. Cooke, J. N. Tu, X. Huang, K. Bibl, and I. Galkin, High voltage antenna-plasma interaction in whistler wave transmission: Plasma sheath effects, $\mathrm{J}$. Geophys. Res., 112, A03205, doi:10.1029/2006JA011683, (2007)

21. Tu, J., P. Song, and B. W. Reinisch, Quasi-static particle simulations of plasma sheath structures around a radio frequency antenna, J. Geophys. Res., 113(A7), A07223, doi:10.1029/2008JA013097, (2008)

22. Chevalier, T. W, U. S. Inan, T. F. Bell, Terminal Impedance and Antenna Current Distribution of a VLF Electric Dipole in the Inner Magnetosphere, IEEE Trans Antenna Propag., 56, 2454, (2008)

23. Song, P, J. Tu, B. W. Reinisch, I. A. Galkin, W. R. Johnston, Whistler Mode Radiation from a Dipole Antenna in Cold Magnetized Plasma, IEEE Trans Antenna Propag., doi: 10.1109/TAP.2021.3121144, (2021)

24. Stenzel, R. L., Whistler modes excited by magnetic antennas: A review, Physics of Plasmas, 26, 080501; doi:10.1063/1.5097852, (2019)

25. Spong, D. A., W.W. Heidbrink, C. Paz-Soldan, X. D. Du, K. E. Thome, M. A. Van Zeeland, C. Collins, A. Lvovskiy, R. A. Moyer, M. E. Austin, D. P. Brennan, C. Liu, E. F. Jaeger, and C. Lau, First direct observation of runaway-electron-driven whistler waves in Tokamaks, Phys. Rev. Lett., 120, 155002, doi:10.1103/PhysRevLett.120.155002, (2018)

26. Scherbarth, M., Smith., D., Adler, A., Stuart, J. \& Ginet, G.P. AFRL's Demonstration and Science Experiments (DSX) mission, Solar Physics and Space Weather Instrumentation III. Edited by Fineschi, Silvano; Fennelly, Judy A. Proc. SPIE, 7438, 74380B (2009).

27. Carpenter, D., and R. Anderson, An ISEE/Whistler model of equatorial electron density in the magnetosphere, J. Geophys. Res., 97(A2), 1097-1108, (1992)

28. Ozhogin, P, P. Song, J. Tu, and B.W. Reinisch, Evaluating the diffusive equilibrium models: Comparison with the IMAGE RPI field-aligned electron density measurements, J. Geophys. Res., 119, 4400-4411, doi:10.1002/2014JA019982, (2014)

29. Paznukhov, V. V., G. S. Sales, K. Bibl, B. W. Reinisch, P. Song, X. Huang, and I. Galkin, Impedance characteristics of an active transmitting antenna radiating in the whistler mode, J. Geophys. Res., 115, A09212, doi:10.1029/2009JA014889, (2010)

30. Reinisch, B. W., et al., The radio plasma imager investigation on the IMAGE spacecraft, Space Sci, Rev., 91, 319-359, (2000)

\section{Figures}




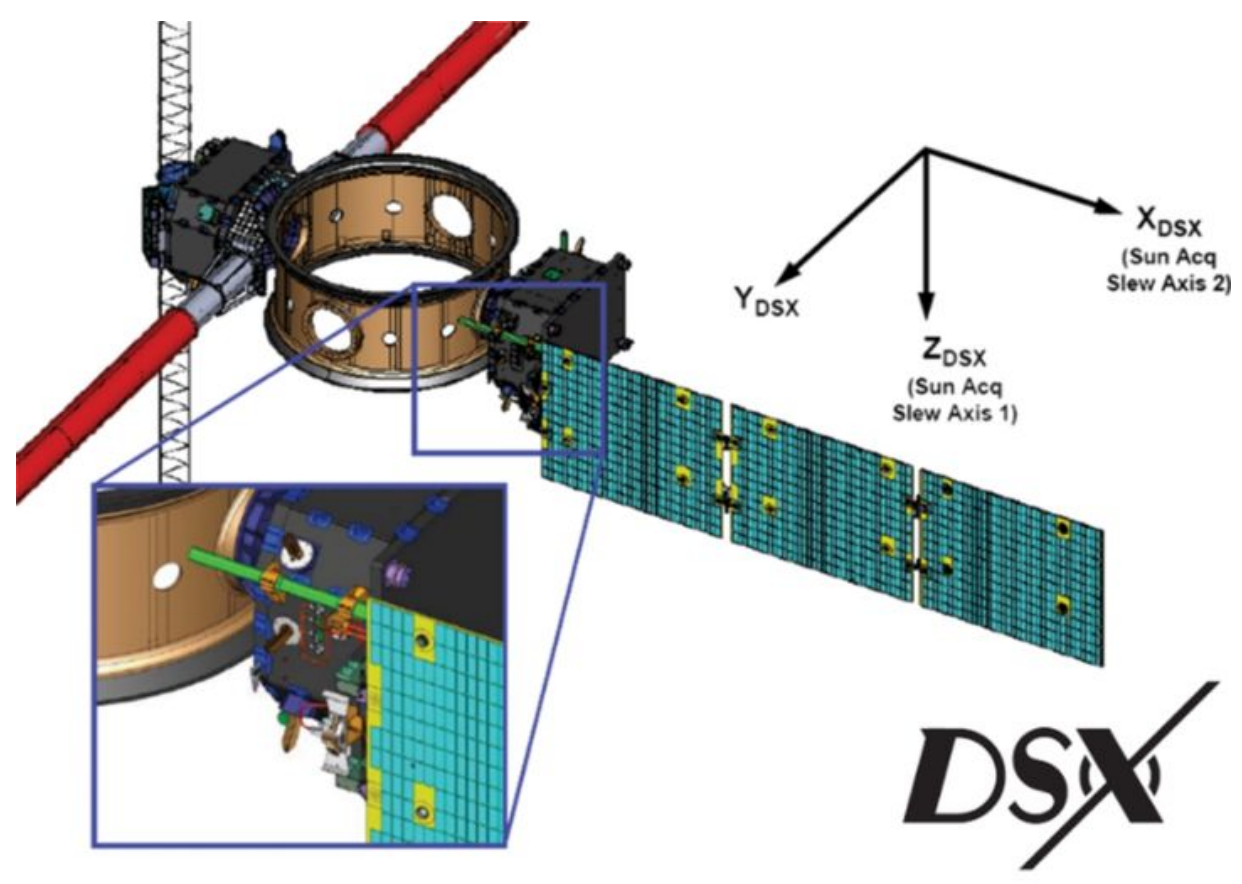

TNT Fundamental Harmonic

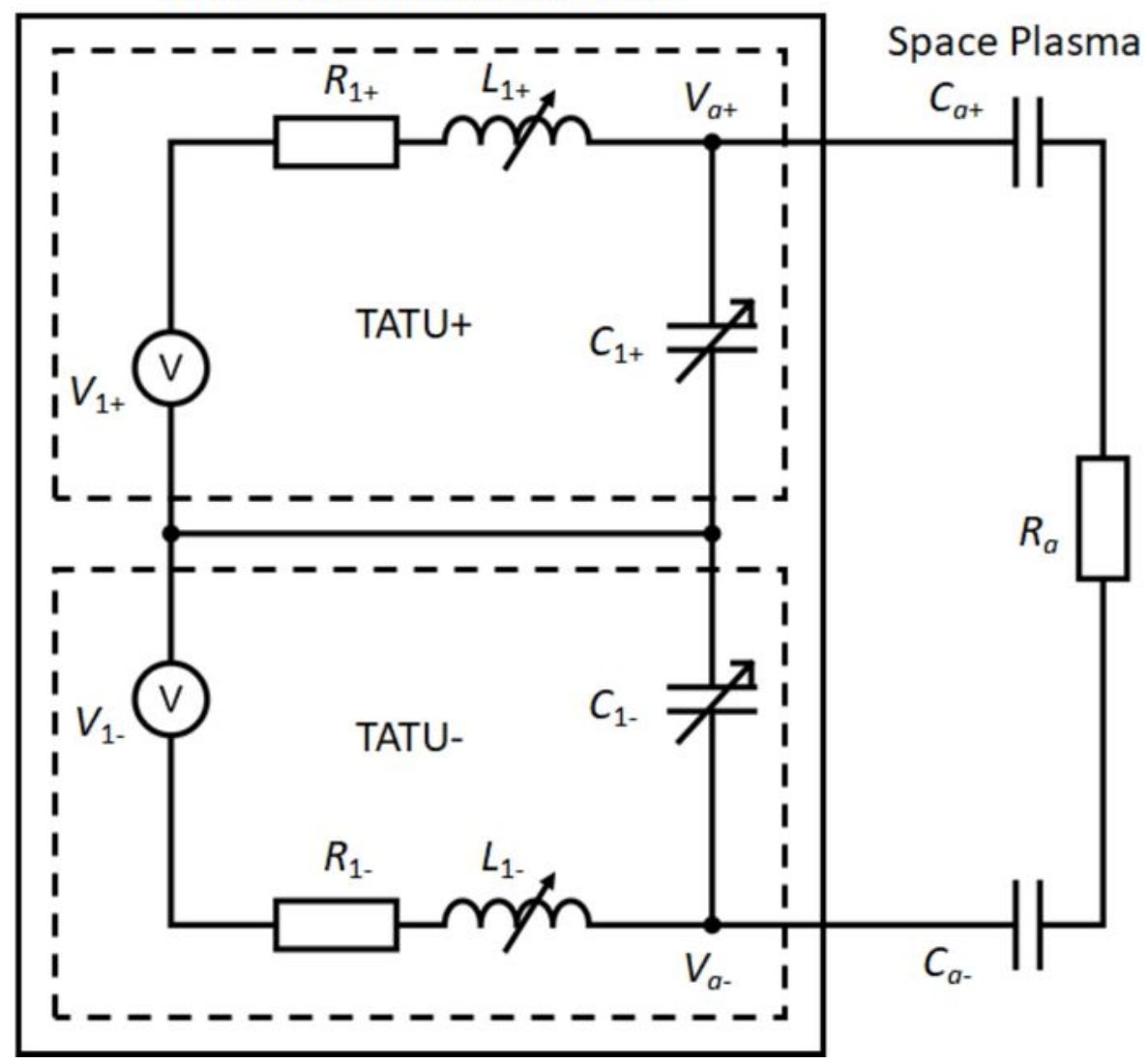

Figure 1

(a)The structure of DSX satellite, https://directory.eoportal.org/web/eoportal/satellitemissions/content/-/article/dsx. The two branches of the TNT antenna are shown as the red cylinders, 40 $\mathrm{m}$ long each side separated by $2 \mathrm{~m}$ diameter satellite body. (b) Equivalent circuit of TNT presented in this report. 


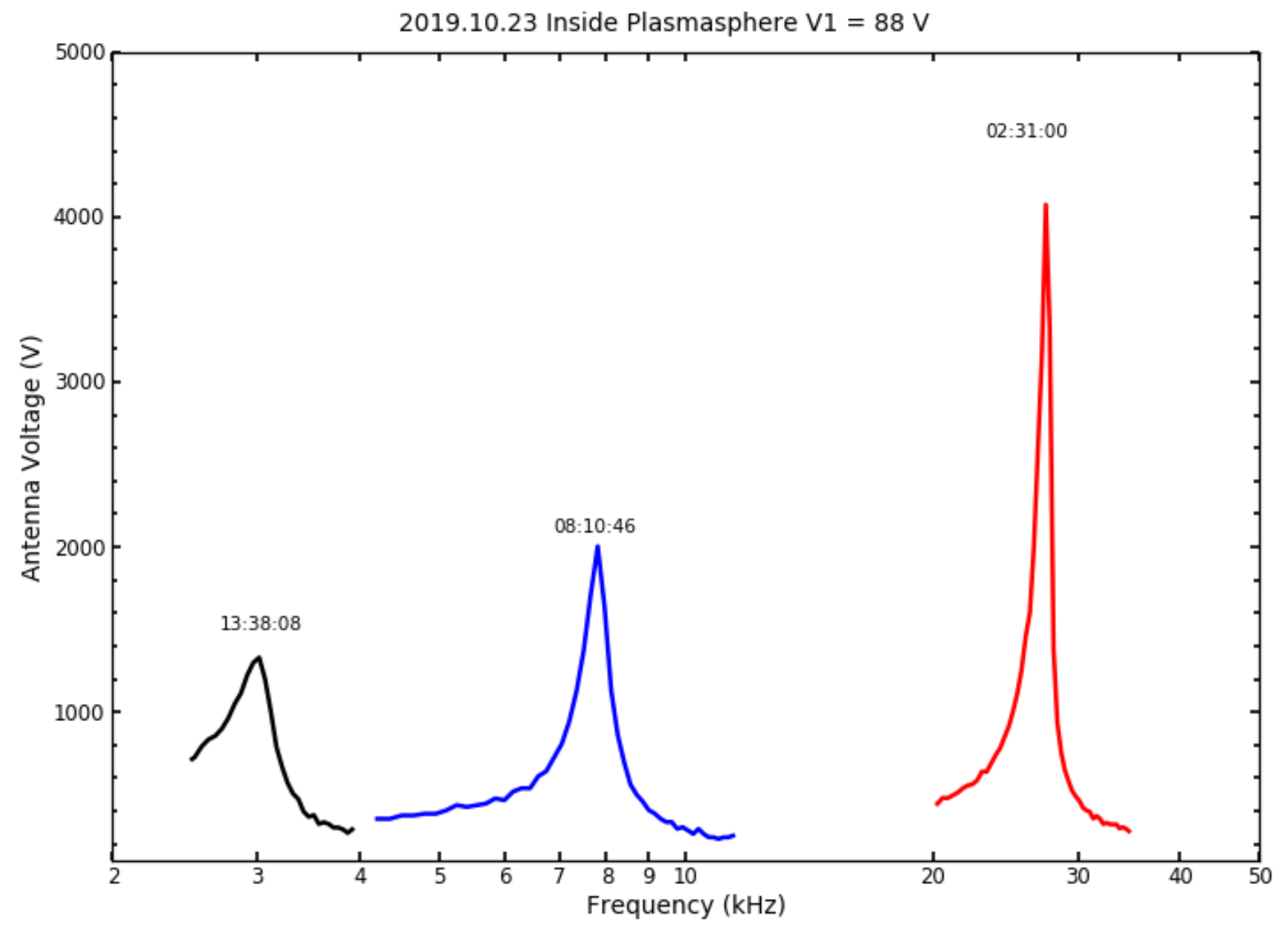

Figure 2

An example of resonance curves, antenna voltage of $\mathrm{Y}+$ branch, $V_{1+}$ as functions of frequency, from highpower transmission $\left(V_{1 \pm}=88 \mathrm{~V}\right)$ in a nominal plasmasphere determined by the Carpenter-Anderson empirical plasmaspheric model ${ }^{27}$. The transmission started in the higher frequency band and then went to mid and lower bands. 

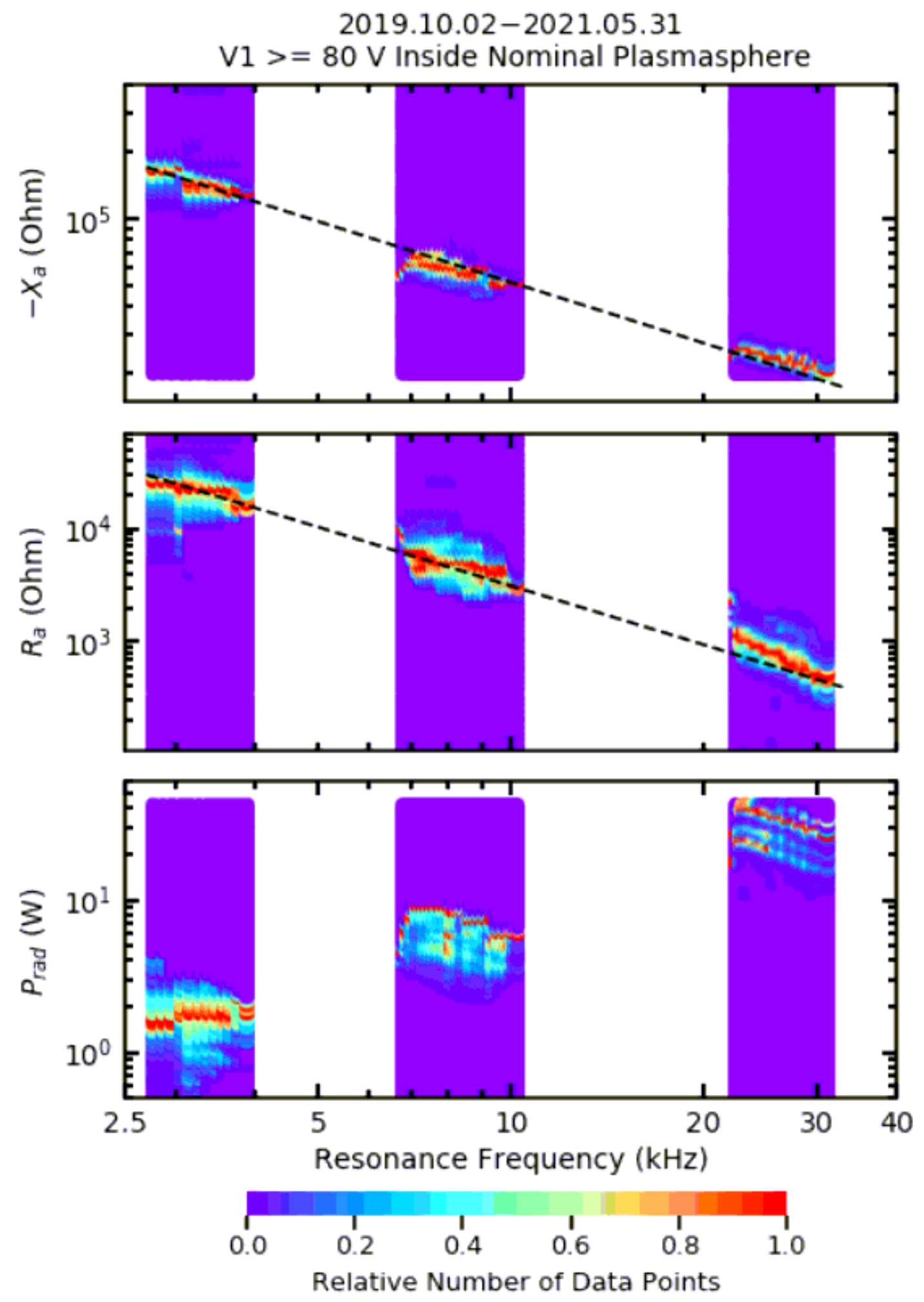

Figure 3

Antenna reactance $-\mathrm{X}_{\mathrm{a}}$ (top row), antenna resistance $\mathrm{R}_{\mathrm{a}}$ (middle row), and antenna power output, $\mathrm{P}_{\text {out }}$ (bottom row). The measurement data are grouped according to the resonance frequency into frequency bins of $100 \mathrm{~Hz}$ wide. The data within each frequency bin are divided according to their logarithmical value of the vertical quantity into 80 cells. The color coding denotes the number of data points within a 
cell divided by the maximum number of data points in any single frequency bin. The total number of data points is about 166,300 .

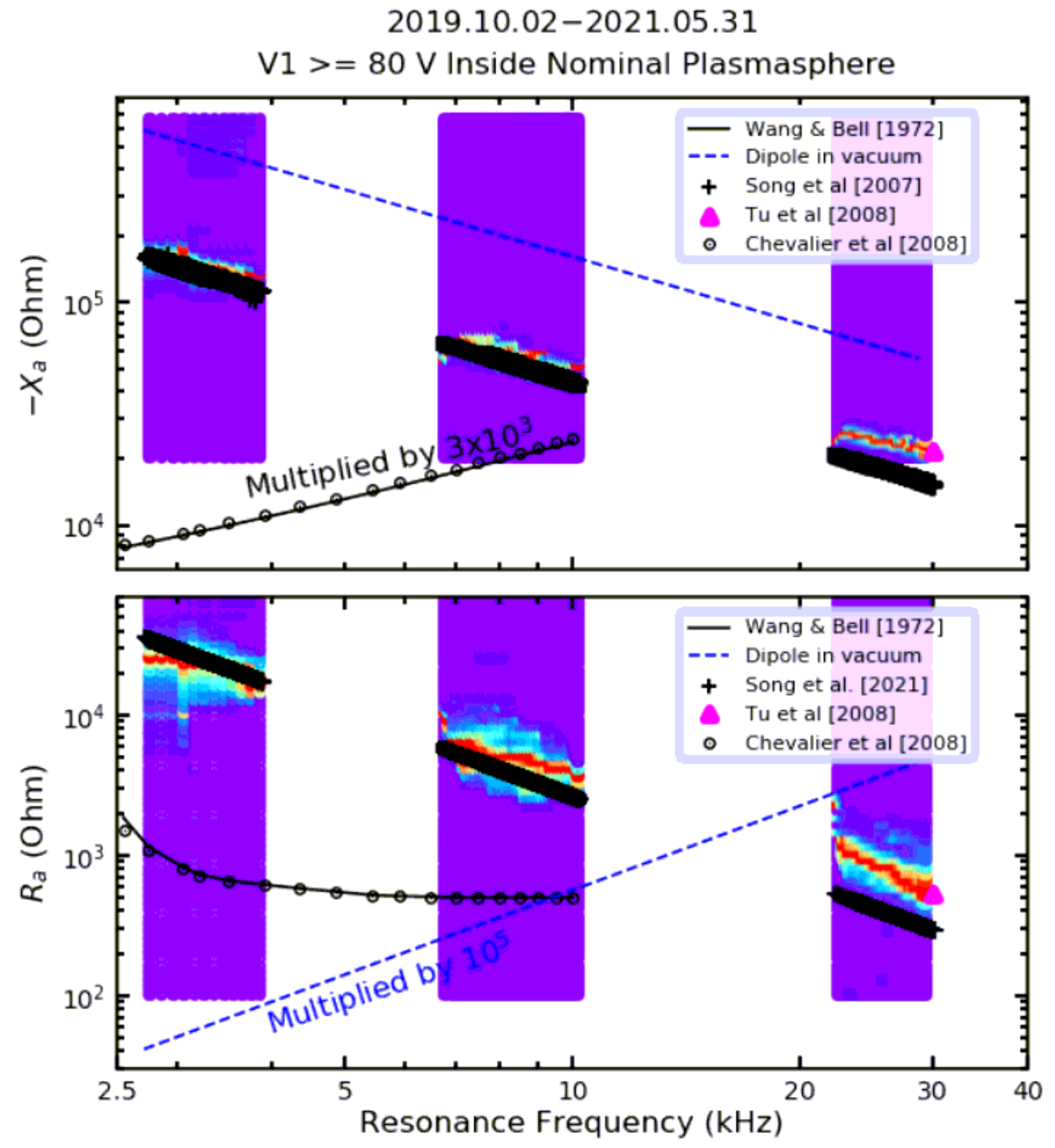

Figure 4

Comparison of DSX results adapted from Figure 3 with the prediction of the radiation impedance in vacuum, blue dashed-lines, Wang and Bell models ${ }^{19}$, black solid lines, Chevalier et al. ${ }^{22}$, black open- 
circles, and Tu et al. simulations ${ }^{21}$, purple solid triangles. Black patches of plus-signs in the upper panel show the antenna reactance predicted by Song et al. antenna sheath model ${ }^{20}$ and those in the lower panel show the radiation resistance predicted by Song et al. whistler radiation model ${ }^{23}$. 